\title{
An exact effective two-qubit gate in a chain of three spins
}

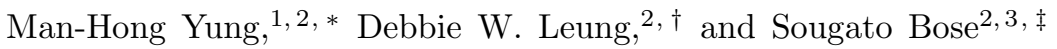 \\ ${ }^{1}$ Physics Department, The Chinese University of Hong Kong, Hong Kong \\ ${ }^{2}$ Institute for Quantum Information, MSC 107-81, \\ California Institute of Technology, Pasadena, CA 91125, USA \\ ${ }^{3}$ Department of Physics and Astronomy, University College London, Gower Street, London WC1E 6BT
}

(Dated: May 27, 2006)

\begin{abstract}
We show that an effective two-qubit gate can be obtained from the free evolution of three spins in a chain with nearest neighbor XY coupling, without local manipulations. This gate acts on the two remote spins and leaves the mediating spin unchanged. It can be used to perfectly transfer an arbitrary quantum state from the first spin to the last spin or to simultaneously communicate one classical bit in each direction. One ebit can be generated in half of the time for state transfer. For longer spin chains, we present methods to create or transfer entanglement between the two end spins in half of the time required for quantum state transfer, given tunable coupling strength and local magnetic field. We also examine imperfect state transfer through a homogeneous XY chain.
\end{abstract}

PACS numbers: 03.67.Mn,03.67.Hk,03.67.-a

\section{INTRODUCTION}

One of the most important resources in quantum information processing is the interaction between different quantum systems, such as those possessed by remote parties or registers of a quantum computer. These systems evolve jointly according to some interaction Hamiltonian. Good methods to convert such interaction into other useful resources, using a well motivated set of control operations, are of paramount importance.

A common framework for studying such conversions involves a system of $n$ qubits (or higher dimensional registers) with unlimited local control. Sophisticated methods for various tasks were developed. For example, in a system coupled by simultaneous arbitrary pairwise interactions, one can extract the interaction on a select pair of qubits 1], which can be used to provide a coupling gate or entanglement. These methods typically approximate the desired task by interspersing the free Hamiltonian evolution with local operations. The required amount of local resources can be reflected by the frequency of manipulations, and an error parameter is given by the interval between them. In general, the task is approximated to first order of this time interval, though sometimes the exact task can be performed without excessive local resources (for examples, see [2, 3] for logic gate construction, and [4, 5, 6] for state transfer and entanglement generation).

In this paper, we consider a simple but nontrivial system in which several tasks can be accomplished perfectly almost without local manipulations. We consider a spin chain with nearest neighbor coupling; the two end spins are data qubits and other spins are mediating the indirect coupling between the data qubits. Furthermore, we

\footnotetext{
*email: mhyung@phy.cuhk.edu.hk

† email: wcleung@caltech.edu

¥email: sougato.bose@qubit.org
}

restrict local control to the data qubits only. The Hamiltonian is given by

$$
H=\sum_{\langle i, j\rangle} \omega_{i j}\left(\sigma_{+i} \sigma_{-j}+\sigma_{-i} \sigma_{+j}\right),
$$

where $\sigma_{+}=\left(\begin{array}{ll}0 & 0 \\ 1 & 0\end{array}\right)$ and $\sigma_{-}=\left(\begin{array}{ll}0 & 1 \\ 0 & 0\end{array}\right)$ are the raising and lowering operators respectively, $i, j$ are spin labels; subscripts of operators indicate the spins being acted on, and the sum is over neighboring spin-pairs denoted by $\langle i, j\rangle$. Expressing $\sigma_{ \pm}$in terms of the Pauli matrices $\left\{\sigma_{x, y, z}\right\}$, we have $\sigma_{ \pm}=\frac{1}{2}\left(\sigma_{x} \pm i \sigma_{y}\right)$ and

$$
H=\sum_{\langle i, j\rangle} \frac{\omega_{i j}}{2}\left(\sigma_{x i} \sigma_{x j}+\sigma_{y i} \sigma_{y j}\right) .
$$

Our main result is for the case of a 3 -spin chain (Fig 1).

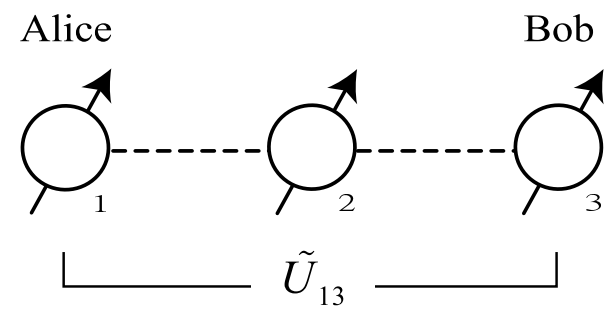

FIG. 1: A spin chain of three spin particles are interacting under the Hamiltonian in (4). An effective two-qubit gate, $\tilde{U}_{13}$, can be obtained at $t=\frac{\pi}{\sqrt{2} \omega}$, when $\omega=\lambda$. The gate can perform perfect state transfer from spin 1 to 3 and also entangle spin 1 with spin 3 , leaving spin 2 unentangled.

We show how to turn the free evolution into an exact effective quantum gate acting on the two data qubits without affecting the mediating spin. This gate can be used to transfer an unknown quantum state between the data spins, to create a maximally entangled state, and 
to communicate classical information. We also explore applications and extensions to this simple basic result.

\section{A. Related works}

Before presenting our main result, we discuss some related works. Our work is motivated by [4, 5] that study quantum state transfer and entanglement distribution in a spin chain of $N$ spins under the free evolution according to the Hamiltonian

$$
H_{G}=-\frac{J}{2} \sum_{\langle i, j\rangle} \vec{\sigma}_{i} \cdot \vec{\sigma}_{j}-B \sum_{i} \sigma_{z i}
$$

where $\vec{\sigma}_{i} \cdot \vec{\sigma}_{j} \equiv \sigma_{x i} \sigma_{x j}+\sigma_{y i} \sigma_{y j}+\sigma_{z i} \sigma_{z j}$. Perfect (near perfect) state transfer between the remotest spins are only allowed for a 4-spin closed (open) chain [4], apart from the trivial case of 2 spins.

Our work is also motivated by [8], which considers a similar 3-spin chain with only nearest neighbor coupling. They found mixed separable initial states such that the two remote spins become entangled by the Hamiltonian without ever entangling the mediating spin. They also proved that such phenomenon is impossible for pure states. In our system, the mediating spin is entangled during the process, but is completely disentangled from the data qubits at the end, leaving the data qubits maximally entangled.

Our work can also find application in the quantum computation scheme in [9]. Reference [9] investigated effective gates between non-neighboring spins in a Heisenberg spin chain (3) with untunable couplings. Our result is an analog in the XY model (1), and has the merit of producing a much simpler two-qubit gate (the composition of a swap gate and a controlled-phase gate) compared to 9$]$.

Time optimal construction of indirect 2-qubit gates, such as SWAP or CNOT, is considered in 7]. Our approach is different in requiring no local manipulations, and also in obtaining an exact implementation of the gate.

During the initial preparation of our manuscript, related works [6] were reported independently by Christandl et al. They considered perfect quantum state transfer over more general spin networks (besides linear chains) under the same Hamiltonian (11). When the interaction strength is homogeneous, i.e. $\omega_{i j}=\omega$, they found that perfect state transfer is possible only between the two ends of an $N=2$ or 3 linear chain, or between the antipoles for a hypercube. When the coupling strength can be suitably tuned, perfect state transfer is possible for any linear chain. Extensions to a Heisenberg chain (3) was considered when a tunable, local magnetic field can be applied to each spin. Our motivating scheme in Sec. II coincides with the $N=3$ case of [6], which also motivated some extensions in Sec. III for longer chains. Our work differs in explicitly considering the evolution on all three spins (instead of a subspace relevant to quantum state transfer as considered in [6]). We exploit different initial configurations that result in more flexibility and better efficiencies in tasks other than state transfer, beyond immediate implications of the ability to perform state transfer.

\section{AN EFFECTIVE TWO-QUBIT GATE}

\section{A. Motivating scheme}

The Hamiltonian for our 3-spin chain (1) is simply

$H=\omega\left(\sigma_{+1} \sigma_{-2}+\sigma_{-1} \sigma_{+2}\right)+\lambda\left(\sigma_{+2} \sigma_{-3}+\sigma_{-2} \sigma_{+3}\right)$.

We first motivate our result by considering quantum state transfer, say, from the first to the last spin:

$$
|\varphi\rangle_{1}|0\rangle_{2}|0\rangle_{3} \rightarrow|0\rangle_{1}|0\rangle_{2}|\varphi\rangle_{3}
$$

Suppose the initial state of the first spin is $|\varphi\rangle_{1}=\alpha|0\rangle_{1}+$ $\beta|1\rangle_{1}$, where $|\alpha|^{2}+|\beta|^{2}=1$. The initial state of the three spins can be written in the form,

$$
\begin{aligned}
|\psi(0)\rangle & =\left(\alpha|0\rangle_{1}+\beta|1\rangle_{1}\right)|0\rangle_{2}|0\rangle_{3} \\
& =\alpha|000\rangle+\beta|100\rangle
\end{aligned}
$$

The state will evolve, under the Hamiltonian (4), as

$$
|\psi(t)\rangle=\alpha|000\rangle+\beta \exp (-i H t)|100\rangle,
$$

where the first term is not affected by the Hamiltonian because $H|000\rangle=0$. The second term can be evaluated analytically. To do this, we make use of the special properties of the raising and lowering operators to obtain

$$
\begin{aligned}
& H|100\rangle=\omega|010\rangle, \\
& H|010\rangle=\omega|100\rangle+\lambda|001\rangle, \\
& H|001\rangle=\lambda|010\rangle .
\end{aligned}
$$

Using (8), the Taylor series expansion can be evaluated explicitly

$$
\begin{gathered}
\exp (-i H t)|100\rangle=\left(1+\frac{\omega^{2}}{\gamma^{2}}(\cos \gamma t-1)\right)|100\rangle \\
\quad+\left(\frac{\omega \lambda}{\gamma^{2}}(\cos \gamma t-1)\right)|001\rangle-i \frac{\omega}{\gamma} \sin \gamma t|010\rangle
\end{gathered}
$$

where $\gamma=\sqrt{\omega^{2}+\lambda^{2}}$. When $\lambda=\omega$ and $t=\tau \equiv \frac{\pi}{\sqrt{2} \omega}$, we have

$$
\exp (-i H \tau)|100\rangle=-|001\rangle
$$

and

$$
\begin{aligned}
|\psi(\tau)\rangle & =\alpha|000\rangle-\beta|100\rangle \\
& =|0\rangle_{1}|0\rangle_{2}\left(\alpha|0\rangle_{3}-\beta|1\rangle_{3}\right) .
\end{aligned}
$$

Perfect state transfer (5) is then obtained by applying the local unitary operator $\sigma_{z 3}$ to the last spin. 


\section{B. The effective two-qubit gate}

To obtain our key result and to better understand the above scheme, we examine the full matrix representation of the unitary operator $U \equiv \exp (-i H \tau)$, with the basis ordered as $|000\rangle,|001\rangle,|100\rangle,|101\rangle,|010\rangle,|011\rangle,|110\rangle$, $|111\rangle$,

$$
U=\left(\begin{array}{cccccccc}
1 & 0 & 0 & 0 & 0 & 0 & 0 & 0 \\
0 & 0 & -1 & 0 & 0 & 0 & 0 & 0 \\
0 & -1 & 0 & 0 & 0 & 0 & 0 & 0 \\
0 & 0 & 0 & -1 & 0 & 0 & 0 & 0 \\
0 & 0 & 0 & 0 & -1 & 0 & 0 & 0 \\
0 & 0 & 0 & 0 & 0 & 0 & -1 & 0 \\
0 & 0 & 0 & 0 & 0 & -1 & 0 & 0 \\
0 & 0 & 0 & 0 & 0 & 0 & 0 & 1
\end{array}\right)
$$

The subspaces spanned by $|0\rangle_{2}$ and $|1\rangle_{2}$ are invariant under $U$. Thus restricting $U$ to either subspace forms an effective two-qubit gate for the remote spins 1 and 3 . In particular, we can consider the restriction $\tilde{U}$ of $U$ to the " $|0\rangle_{2}$ subspace,"

$$
\tilde{U}_{13}=\left(\begin{array}{cccc}
1 & 0 & 0 & 0 \\
0 & 0 & -1 & 0 \\
0 & -1 & 0 & 0 \\
0 & 0 & 0 & -1
\end{array}\right)
$$

which is a composition of the SWAP gate and a "jointphase-gate" $\operatorname{Diag}(1,-1,-1,-1)$ that is equivalent to the controlled $-\sigma_{z}$ acting on spins 1 and 3 (up to renaming of basis states and an overall sign).

In the scheme discussed in Sec. IA spin 3 is initialized as $|0\rangle_{3}$; the effect of the joint-phase-gate can be reverted by the local $\sigma_{z 3}$, so that the net effect is to swap spins 1 and 3 , resulting in perfect state transfer.

\section{State transfer using $\tilde{U}_{13}$}

Equation (13) shows that perfect state transfer (up to an irrelevant overall "-" sign) can be performed without the final local gate $\sigma_{z 3}$, if spin 2 and spin 3 are initialized to be $|0\rangle_{2}|1\rangle_{3}$ instead of $|0\rangle_{2}|0\rangle_{3}$. (Alternatively, we can also initialize them to be $|1\rangle_{2}|0\rangle_{3}$.) Thus, barring initialization of spins 2 and 3, no local manipulation is required at all! As a final remark, state transfer due to (13) is coherent, thus it is entanglement-preserving and in particular, $|\phi\rangle_{a 1}|01\rangle_{23} \rightarrow-|10\rangle_{12}|\phi\rangle_{a 3}$ for any joint state $|\phi\rangle_{a 1}$ on spin 1 and an ancillary system $a$ of arbitrary dimension.

\section{Classical communication and entanglement generation}

The ability to transfer a certain amount of quantum information from one end of a spin chain to another obvi- ously implies the ability to transfer at least as much classical communication, and to share at least as much entanglement (in units of ebit) between the two ends when the transfer is entanglement preserving. In this section, we present communication and entanglement sharing or generation schemes (they differ by whether entanglement is distributed or created) that outperform the above obvious methods.

Equation (13) suggests a simple method for bidirectional classical communication [10] between spins 1 and 3. Suppose spins 1 and 3 are in the possession of Alice and Bob respectively. Spin 2 is in the control of the communication company who initializes the spin to $|0\rangle_{2}$ but does nothing otherwise. Since $\tilde{U}$ acts exactly as a swAP gate on classical states, Alice and Bob can each send one classical bit to the other. Note that the combined communication rate is twice the rate of quantum state transfer and that the qualitative ability to communicate simultaneously in both direction is not implied from the ability to perform quantum state transfer.

Equation (13) also suggests an obvious method for creating entanglement between spins 1 and 3 - the gate $\tilde{U}$ creates one ebit starting from the initial product state $|+\rangle_{1}|+\rangle_{3}$, where $| \pm\rangle=\frac{1}{\sqrt{2}}(|0\rangle \pm|1\rangle)$. As $\tilde{U}$ acts like the SWAP gate followed by the joint-phase-gate, the sWAP leaves the state invariant, and the joint-phase-gate takes $|+\rangle_{1}|+\rangle_{3}$ to $\frac{1}{\sqrt{2}}\left(|0\rangle_{1}|-\rangle_{3}-|1\rangle_{1}|+\rangle_{3}\right)$, which is maximally entangled. Spin 2 is invariant under $U$ and is disentangled from spins 1 and 3 at the end. Finally, $\tilde{U}$ can be used to share two ebits if applied to an initial state $|\Phi\rangle_{a 1} \otimes|\Phi\rangle_{3 b}$ where $|\Phi\rangle=\frac{1}{\sqrt{2}}(|00\rangle+|11\rangle)$ and $1, a$ and $3, b$ are possessed by Alice and Bob respectively.

The above methods are optimal if $\tilde{U}$ is a fixed given resource and they require no local control besides local state initialization. Exactly one of the SWAP and the phase-gate components of $\tilde{U}$ are used in each protocol.

More generally, given the Hamiltonian $H$, there are alternative schemes for the various tasks that do not involve implementing $\tilde{U}$ and are more efficient. For example, let $\sqrt{U} \equiv \exp \left(-i H \frac{\tau}{2}\right)$, which can be written explicitly (again in the basis $|000\rangle,|001\rangle,|100\rangle,|101\rangle,|010\rangle$, $|011\rangle,|110\rangle,|111\rangle)$ as

$$
\sqrt{U}=\left(\begin{array}{cccccccc}
1 & 0 & 0 & 0 & 0 & 0 & 0 & 0 \\
0 & \frac{1}{2} & -\frac{1}{2} & 0 & \frac{-i}{\sqrt{2}} & 0 & 0 & 0 \\
0 & -\frac{1}{2} & \frac{1}{2} & 0 & \frac{-i}{\sqrt{2}} & 0 & 0 & 0 \\
0 & 0 & 0 & 0 & 0 & \frac{-i}{\sqrt{2}} & \frac{-i}{\sqrt{2}} & 0 \\
0 & \frac{-i}{\sqrt{2}} & \frac{-i}{\sqrt{2}} & 0 & 0 & 0 & 0 & 0 \\
0 & 0 & 0 & \frac{-i}{\sqrt{2}} & 0 & \frac{1}{2} & -\frac{1}{2} & 0 \\
0 & 0 & 0 & \frac{-i}{\sqrt{2}} & 0 & -\frac{1}{2} & \frac{1}{2} & 0 \\
0 & 0 & 0 & 0 & 0 & 0 & 0 & 1
\end{array}\right) .
$$

The fourth and the fifth columns imply that

$$
\begin{aligned}
& \sqrt{U}|0\rangle_{2}|11\rangle_{13}=-i|1\rangle_{2} \frac{1}{\sqrt{2}}(|01\rangle+|10\rangle)_{13} \\
& \sqrt{U}|1\rangle_{2}|00\rangle_{13}=-i|0\rangle_{2} \frac{1}{\sqrt{2}}(|10\rangle+|01\rangle)_{13} .
\end{aligned}
$$


Applying $H$ for a period of time $\frac{\tau}{2}$, half of that required for implementing $\tilde{U}$, one can create a maximally entangled state from a product state, without ancillas, and with spin 2 disentangled at the end.

More amusingly, Alice and Bob can repeat the procedure indefinitely without resetting the mediating spin: Let spin 2 be in $|0\rangle_{2}$ initially. Alice and Bob input the states $|1\rangle_{1}|1\rangle_{3}$, apply $H$ for time $\frac{\tau}{2}$, extract the maximally entangled state (using (15)), and then input the state $|0\rangle_{1}|0\rangle_{3}$ instead. By now, spin 2 has evolved to $|1\rangle_{2}$, and according to (16), another maximally entangled state can be created after time $\frac{\tau}{2}$, and so on. This requires only one initialization of the mediating spin and the ability to prepare $|0\rangle$ and $|1\rangle$ locally by Alice and Bob, and to input/output a quantum state of the data qubits at intervals separated by $\frac{\tau}{2}$.

\section{E. Effective two-qubit gate in a network}

With the spin chain in Fig. 11 as a basic unit, an effective two-qubit gate can be obtained in a larger network of spins. For example, consider a network (see Fig. 2) with three different homogeneous chains, evolved by a Hamiltonian $H_{3}=H_{a}+H_{b}+H_{c}$ where each of $H_{a, b, c}$ acts on one chain and has the form of (4), and $\omega_{a}=\lambda_{a}, \omega_{b}=\lambda_{b}$, and $\omega_{c}=\lambda_{c}$, but $\omega_{a}, \omega_{b}$ and $\omega_{c}$ can be different. Let $|\tilde{0}\rangle_{2} \equiv|0\rangle_{2 a}|0\rangle_{2 b}|0\rangle_{2 c}$, and $|\tilde{1}\rangle_{2} \equiv\left(\omega_{a}|1\rangle_{2 a}+\omega_{b}|1\rangle_{2 b}+\omega_{c}|1\rangle_{2 c}\right) / \omega$, where we redefine $\omega \equiv\left(\omega_{a}^{2}+\omega_{b}^{2}+\omega_{c}^{2}\right)^{1 / 2}$. The joint evolution is then analogous to the single chain case, and an effective two-qubit gate $\tilde{U}_{13}$ in (13) can be obtained at $t=\tau$. Similar argument applies to any number of chains. Each extra chain simply increases the coupling strength. We note that similar techniques has been used in the context of quantum walk, see for example [12].

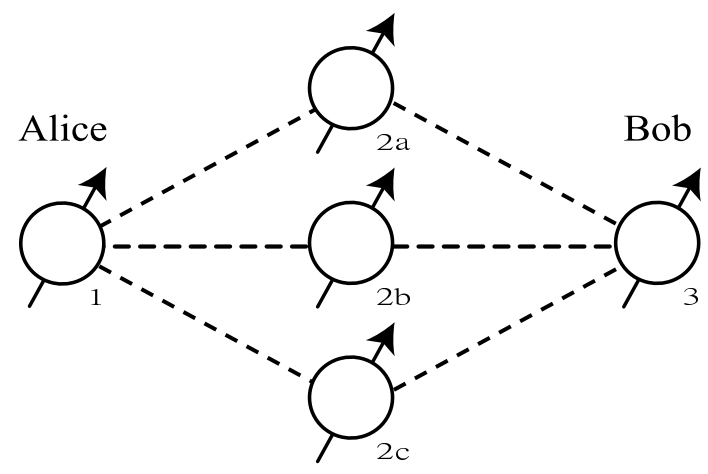

FIG. 2: A network of spins consists of three spin chains in Fig. 11 An effective two-qubit gate, $\tilde{U}_{13}$, can be obtained even if the coupling strength for the chains are different.

\section{SPIN CHAINS WITH $N>3$ SPINS}

In this section, we extend the above results to spin chains with $N>3$ spins. We first consider generating or sharing entanglement in inhomogeneous chains and then consider quantum state transfer for homogeneous chains.

\section{A. Inhomogeneous chains}

We first consider inhomogeneous chains in which the coupling strength $\omega_{j+1}$ can be tuned for different pairs of spins. Our entanglement generating or sharing schemes borrow techniques from Christandl et al [6]. Our scheme takes about half of the time required for quantum state transfer.

Consider a chain of $N$ spins. We want to prepare a maximally entangled state $\frac{1}{\sqrt{2}}\left(|0\rangle_{1}|1\rangle_{N}+|1\rangle_{1}|0\rangle_{N}\right)$ between the data spins 1 and $N$ at the two ends. When $N$ is odd, we find an entanglement generating scheme with a simple initial spin configuration. When $N$ is even, we find an entanglement sharing scheme that requires preexisting entanglement in the chain and a local magnetic field. We are going to discuss these two cases separately.

\section{Odd number of spins}

We use a more convenient basis defined as $\equiv$ $\frac{1}{\sqrt{2}}(|100 \cdots\rangle+|\cdots 001\rangle),|\tilde{2}\rangle \equiv \frac{1}{\sqrt{2}}(|010 \cdots\rangle+|\cdots 010\rangle)$, and similarly for $|\tilde{j}\rangle, j=1, \cdots, n-1$, and $|\tilde{n}\rangle \equiv$ $|0 \cdots 010 \cdots 0\rangle$. Here, $n=\frac{1}{2}(N+1)$ is the position of the middle spin. Suppose the initial state is $|\tilde{n}\rangle$. Then, Hamiltonian in (2) acts in the basis $|\tilde{1}\rangle,|\tilde{2}\rangle, \cdots,|\tilde{n}\rangle$ as

$$
H_{\text {odd }}=\left(\begin{array}{ccccc}
0 & \omega_{12} & 0 & \cdots & 0 \\
\omega_{12} & 0 & \omega_{23} & \cdots & 0 \\
0 & \omega_{23} & 0 & \cdots & \vdots \\
\vdots & \vdots & \vdots & \ddots & \sqrt{2} \omega_{n-1 n} \\
0 & 0 & \cdots & \sqrt{2} \omega_{n-1 n} & 0
\end{array}\right) .
$$

The task to generate entanglement is the same as to rotate $|\tilde{n}\rangle$ into $|\tilde{1}\rangle$, and it is equivalent to transferring a quantum state in a chain of length $n=\frac{1}{2}(N+1)$ as solved by Christandl et al $[\underline{6}]$. When the matrix elements $\omega_{j, j+1}$ are chosen such that

$$
\begin{gathered}
\omega_{j, j+1} \propto \begin{cases}\sqrt{j(n-j)}, & \text { for } j \neq n-1, \\
\sqrt{n-1} / \sqrt{2}, & \text { for } j=n-1,\end{cases} \\
\left|\left\langle\tilde{1}\left|\exp \left(-i H_{\text {odd }} t\right)\right| \tilde{n}\right\rangle\right|=1
\end{gathered}
$$

when $t=\pi / \lambda$ for a constant $\lambda$ described in [6]. This concludes the entanglement generation scheme.

Note that the time for the above scheme is constant, while the coupling strengths $\omega_{j, j+1}$ is a function of $n$ 
(and most are roughly proportional to $n$ ). The resource in this framework can be identified as $\omega_{j, j+1} t$ or simply $\omega_{j, j+1}$. Thus, our scheme creates entanglement using roughly half of the resource needed in the obvious method of entanglement sharing via quantum state transfer.

\section{Even number of spins}

We describe a similar scheme for an even number of spins, which requires an initially entangled state, and a time independent local magnetic field. (It is thus an entanglement sharing scheme.) Let $|\tilde{j}\rangle$ be as defined before, except now $|\tilde{n}\rangle \equiv \frac{1}{\sqrt{2}}(|0 \cdots 10 \cdots 0\rangle+|0 \cdots 01 \cdots 0\rangle)$. Again, we initialize our state to $|\tilde{n}\rangle$, and entanglement sharing is achieved by rotating $|\tilde{n}\rangle$ to $|\tilde{1}\rangle$. The nontrivial part of the Hamiltonian in this basis is

$$
H_{\text {even }}=\left(\begin{array}{ccccc}
0 & \omega_{12} & 0 & \cdots & 0 \\
\omega_{12} & 0 & \omega_{23} & \cdots & 0 \\
0 & \omega_{23} & 0 & \cdots & 0 \\
\vdots & \vdots & \vdots & \ddots & \omega_{n-1 n} \\
0 & 0 & \cdots & \omega_{n-1 n} & \omega_{n n+1}
\end{array}\right)
$$

$H_{\text {even }}$ differs from $H_{\text {odd }}$ only in the extra term $\omega_{n, n+1}$ in the lower right corner. The extra term can be removed by applying a static magnetic field of strength $\frac{1}{2} \omega_{n, n+1}$ to the middle two spins. Mathematically, this adds a local Hamiltonian $H_{B}=-\frac{1}{2} \omega_{n, n+1}\left(\sigma_{z, n}+\sigma_{z, n+1}\right)$, and since $H_{B}|\tilde{n}\rangle=0, H_{B}|\tilde{j}\rangle=\omega_{n, n+1}|\tilde{j}\rangle$ for all $j<n$, the diagonal terms in $H_{\text {even }}+H_{B}$ are all equal to $\omega_{n, n+1}$ and contribute an irrelevant phase only. The rest follows from the odd case.

As a side remark, for a homogeneous chain when the coupling constants $\omega_{i j}$ are equal, an even chain entangles slightly better. The two extra factors of $\sqrt{2}$ in $H_{\text {odd }}$ causes perfect entanglement to be possible only for $N=$ 3 , while it is possible for $N=4$ and 6 in the even case.

\section{B. Homogeneous chains}

For homogeneous spin chains, we study the fidelity of quantum state transfer

$$
|\varphi\rangle_{1}|0\rangle_{2} \cdots|0\rangle_{N} \rightarrow|0\rangle_{1}|0\rangle_{2} \cdots|\varphi\rangle_{N} .
$$

where the fidelity (averaged over inputs) [4] is defined as

$$
\begin{aligned}
F & =\frac{f(t)}{3}+\frac{f(t)^{2}}{6}+\frac{1}{2}, \text { where } \\
f(t) & \equiv|\langle 10 \cdots 0|\exp (-i H t)| 0 \cdots 01\rangle|
\end{aligned}
$$

and a maximization over $t$ is then taken.

In Fig. 3] we plotted numerical maximization of $f(t)$ over a sufficient long but finite range of $t$. A comparison with similar numerical maximization for the Heisenberg chain (with Hamiltonian given by (3)) suggests that the $\mathrm{XY}$ chain (with Hamiltonian given by (1) is more effective in quantum state transfer.

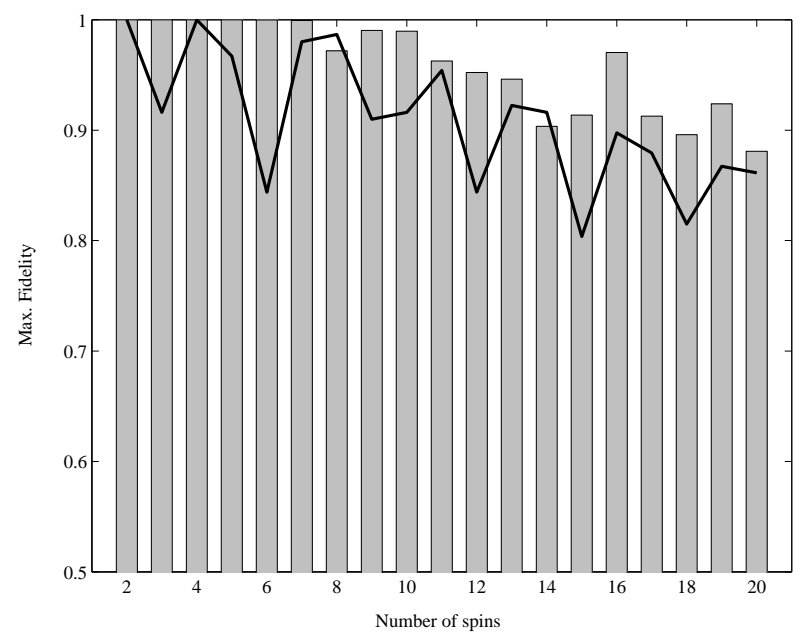

FIG. 3: Average fidelity $f(t)$, maximized over $t \in(0,2000 / \omega)$ in (22), versus the number of spins $N$ in the chain. The bar chart and the line are for the XY (11) and Heisenberg (3) chains respectively.

For asymptotically large $N$, using an expression for $f(t)$ in Ref. [6] for the XY chain,

$$
f(t)=\left|\frac{2}{N+1} \sum_{m=1}^{N} \sin \frac{\pi m}{N+1} \sin \frac{\pi m N}{N+1} e^{-i E_{m} t}\right|,
$$

where $E_{m}=-2 \omega \cos \frac{m \pi}{N+1}$. Let $J_{k}$ be the Bessel functions. The Jacobi-Anger expansion [13, 14] states that

$$
e^{i z \cos \theta}=\sum_{k=-\infty}^{\infty} i^{k} J_{k}(z) e^{-i k \theta} .
$$

Applying this to (24), taking $N$ to be large and $t_{0}$ to be of order $N$, and neglecting the terms with $k \gg N$ or $k \ll N$, we have,

$$
f\left(t_{0}\right) \approx 2\left|J_{N}\left(2 \omega t_{0}\right)+J_{N+2}\left(2 \omega t_{0}\right)\right| .
$$

Since $N+2+\xi(N+2)^{1 / 3} \approx N+\xi N^{1 / 3}$ for all $\xi$, and $J_{N}\left(N+\xi N^{1 / 3}\right) \approx(2 / N)^{1 / 3} A\left(-2^{1 / 3} \xi\right)$ [15], where $A(\cdot)$ is the Airy function, applying $2 \omega t_{0}=N+0.8089 N^{1 / 3}$ gives a lower bound on the fidelity

$$
f\left(t_{0}\right) \approx 2.6998 N^{-1 / 3} .
$$

This is twice the value of $f\left(t_{0}\right)$ for a Heisenberg chain (3) for the same $t_{0}[4]$.

Although perfect state transfer is possible only for $N=2$ and $N=3$, fidelities very close to 1 are obtainable for sufficiently long evolution times. Such long evolution times can be shortened using a non-homogeneous 
magnetic field applied to individual spins. For example, for an $N=4 \mathrm{XY}$ chain, the maximum fidelity obtained is 0.99997 at $t=53.4 / \omega$, in the time interval of $(0,100 / \omega)$. However, if we apply a magnetic field of strength $B=0.625 \omega$ on the two middle spins only, the fidelity is 0.99991 at $t=6.25 / \omega$, see Fig. [u
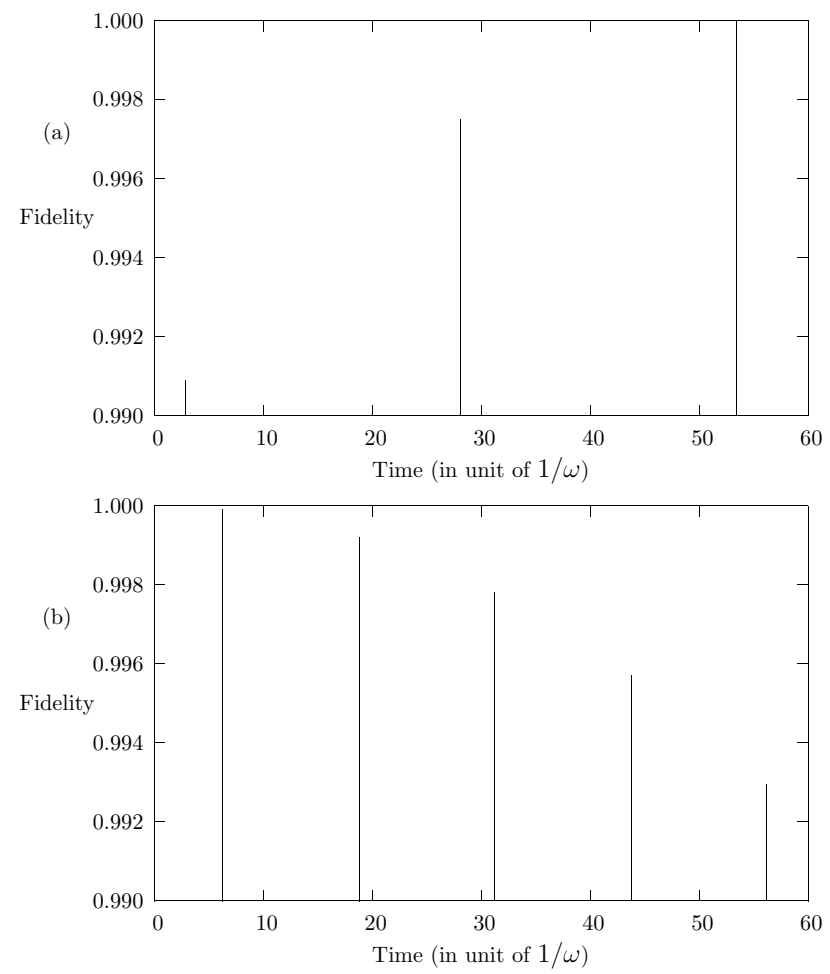

FIG. 4: Peaks of the average fidelity $F(t)$ (22) as a function of time for an $N=4$ spin chain. Plot (a) represents the case without a magnetic field, plot (b) represents the case when the middle two spins are subject to a magnetic field of strength $B=0.625 \omega$. The maximum peak is 0.99991 at $t=6.25 / \omega$.

\section{CONCLUSION}

We have demonstrated how to obtain an exact effective two-qubit gate in a chain of three spins without local manipulations, and discussed various applications of it, including quantum state transfer, classical communication, and entanglement generation and distribution. We also discuss some extensions to longer chains. Other observations of the system can be found in the Appendix.

\section{Acknowledgments}

We thank Andrew Landahl for help comments on an earlier manuscript. This work is partially supported from the US National Science Foundation under grant no. EIA-0086038. DWL thanks the Richard C. Tolman Endowment Fund at Caltech for partial support. SB thanks for a postdoctoral scholarship at the Caltech Institute for Quantum Information, during which this work started.

\section{APPENDIX: MISCELLANEOUS RESULTS}

Here we explore more applications of the gate $U$ and other results. In a chain of three spins, the gate $U$ can actually entangle any pair of spins. For example, to entangle spin 1 and 2, while leaving spin 3 unentangled, we can initialize the state to be $|0\rangle_{1}|+\rangle_{2}|+\rangle_{3}$. The final state is $\frac{1}{\sqrt{2}}\left(|0\rangle_{1}|-\rangle_{2}-|1\rangle_{1}|+\rangle_{2}\right)|0\rangle_{3}$. On the other hand, the gate $U$ can also act as an effective one-qubit gate, $\sigma_{z 2}$, for the middle spin if we initialize the state to be $|0\rangle_{1}\left(\alpha|0\rangle_{2}+\beta|1\rangle_{2}\right)|0\rangle_{3}$, for any $\alpha$ and $\beta$.

Together with an application of a single-qubit rotation, we can readily generate a three-qubit entangled state, the $W$ state [1] , $\frac{1}{\sqrt{3}}(|101\rangle+|011\rangle+|110\rangle)$, by a free evolution under the Hamiltonian in (4). More explicitly, $\exp (-i H t)|101\rangle=\cos \gamma t|101\rangle-i \frac{\omega}{\gamma} \sin \gamma t|011\rangle-$ $i \frac{\lambda}{\gamma} \sin \gamma t|110\rangle$. When $\omega=\lambda$ and $t=\tan ^{-1}(\sqrt{2}) / \sqrt{2} \omega$, we obtain the $W$ state after applying the phase gate $|0\rangle\langle 0|+i| 1\rangle\langle 1|$ to the middle spin.
[1] N. Linden, H. Barjat, R. Carbajo, and R. Freeman, Chem. Phys. Lett. 305 28, (1999); D. W. Leung, I. L. Chuang, F. Yamaguchi, and Y. Yamamoto, Phys. Rev. A 61, 042310 (2000); J. Jones and E. Knill. J. of Mag. Res. 141, 322 (1999); J. L. Dodd, M. A. Nielsen, M. J. Bremner, R. T. Thew, Phys. Rev. A 65, 040301 (R) (2002); P. Wocjan, D. Janzing, Th. Beth, Quant. Inf. Comp. 2, 117 (2002); D. Leung, J. Mod. Opt. 49, 1199 (2002); M. Stollsteimer and G. Mahler, Phys. Rev. A 64, 052301 (2001); N. Khaneja, R. Brockett, and S.
J. Glaser, Phys. Rev. A. 63, 032308 (2001); P. Wocjan, M. Roetteler, D. Janzing, and Th. Beth, Phys. Rev. A 65, 042309 (2002); M. A. Nielsen, M. J. Bremner, J. L. Dodd, A. M. Childs, C. M. Dawson, Phys. Rev. A 66, 022317 (2002).

[2] D. W. Leung, I. L. Chuang, F. Yamaguchi, and Y. Yamamoto. Phys. Rev. A, 61042310 (2000).

[3] J. Jones and E. Knill. J. of Mag. Res., 141 322-5 (1999).

[4] S. Bose, Phys. Rev. Lett 91, 207901 (2003).

[5] V. Subrahmanyam, quan-ph/0307135 
[6] M. Christandl, N. Datta, A. Ekert and A.J. Landahl, quan-ph/0309131

[7] N. Khaneja, S. J. Glaser and R. W. Brockett, Phys. Rev. A, 65, 032301 (2002).

[8] T. S. Cubitt, F. Verstraete, W. Dür and J.I. Cirac, Phys. Rev. Lett. 91, 037902 (2003)

[9] S. C. Benjamin and S. Bose, Phys. Rev. Lett. 90, 247901 (2003).

[10] C. Bennett, A. Harrow, D. Leung, and J. Smolin, IEEE Trans. Inf. Theory, 48580 (2002).
[11] W. Dür, G. Vidal and J. I. Cirac, Phys. Rev. A 62, 062314 (2000).

[12] A. Childs, E. Farhi, J. Goldstone, and S. Gutmann, Quant. Inf. Comp. 2, 181 (2002).

[13] G. Arfken and H. Weber, Mathematical Methods for Physicists (Harcourt, San Diego, 2001).

[14] Weisstein Mathematics, http://mathworld.wolfram.com

[15] M. Abramowitz and I. A. Stegun, Handbook of Mathematical Functions (Dover, New York, 1972). 